\title{
Functional consequences of plant-animal interactions along the mutualism-antagonism gradient
}

\author{
María C. Rodríguez-Rodríguez, Pedro Jordano, and Alfredo Valido ${ }^{1}$ \\ Department of Integrative Ecology, Estación Biológica de Doñana (EBD-CSIC), \\ ClAmerico Vespucio 26, Isla de la Cartuja, 41092, Sevilla, Spain
}

\begin{abstract}
Plant-animal interactions are pivotal for ecosystem functioning, and usually form complex networks involving multiple species of mutualists as well as antagonists. The costs and benefits of these interactions show a strong context-dependency directly related to individual variation in partner identity and differential strength. Yet understanding the context-dependency and functional consequences of mutualistic and antagonistic interactions on individuals remains a lasting challenge. We use a network approach to characterize the individual, plant-based pollination interaction networks of the Canarian Isoplexis canariensis (Plantaginaceae) with a mixed assemblage of vertebrate mutualists (birds and lizards) and invertebrate antagonists (florivores, nectar larcenists, and predispersal seed predators). We identify and quantify interaction typologies based on the sign (mutualistic vs. antagonistic) and strength (weak vs. strong) of animal-mediated pollination and test the relationship with individual female reproductive success (FRS). In addition, we document pollinator movement patterns among individual plants to infer events of pollen transfer/receipt that define the plant mating networks and test the relationship with FRS. We identify six interaction typologies along a mutualism-antagonism gradient, with two typologies being over-represented involving both mutualists and antagonists and influencing FRS. Plants showing strong mutualistic interactions, but also (weak or strong) interactions with antagonists are relatively better connected in the mating network (i.e., with higher potential to transfer or receive pollen). Thus, mixed flower visitor assemblages with mutualists and antagonists give plants increased their importance in the mating networks, promote outcrossing and increasing both female and male fitness. Our approach helps characterize plant-animal interaction typologies, the context-specificity of diversified mutualisms, and a better forecasting of their functional consequences.
\end{abstract}

Key words: antagonist; bird pollination; Canary Islands; female reproductive success; individual-based pollination networks; interaction strength; Isoplexis canariensis; mating network; mutualist.

\section{INTRODUCTION}

Mutualistic plant-animal interactions are pivotal elements of the structure and dynamics of ecological communities (Bascompte and Jordano 2014, Kissling and Schleuning 2015). Unveiling the complexity of these interactions is a challenging task: each plant species frequently interacts with multiple mutualistic agents, and partner identities and their interaction strengths continually change over time and space (Thompson 2005). Recent research has examined not only the structural properties of these complex patterns of interaction, but also their ecological and evolutionary consequences for whole communities (Bascompte and Jordano 2014, and references therein). An outstanding issue is to understand how these multispecies networks, and their functional consequences, emerge from the structure of mutualistic interactions within populations and, in turn, from the heterogeneous distribution of interactions among individual plants and

Manuscript received 23 March 2016; revised 17 January 2017; accepted 24 January 2017. Corresponding Editor: Neal M. Williams.

${ }^{1}$ Corresponding Author. E-mail: avalido@ebd.csic.es animals (i.e., individual-based interaction networks; Dupont et al. 2011, Gómez et al. 2011, Dupont et al. 2014, Tur et al. 2014, Valverde et al. 2016). This variation and its functional consequences constitutes the raw material for coevolution (Thompson 2005).

Recent studies of pollination (Fortuna et al. 2008, Dupont et al. 2011, 2014, Gómez et al. 2011, Gómez and Perfectti 2012, Tur et al. 2014, Valverde et al. 2016), seed dispersal (Cantor et al. 2013) and ant-plant interactions (Dáttilo et al. 2014) have begun to unravel how biotic interactions are organized at the scale of individual partners. These studies largely examine a simplified interaction typology to include plants and their mutualistic partners. However, antagonistic partners can radically alter the outcomes of mutualistic interactions (Irwin and Brody 1998, Bronstein et al. 2003, Irwin 2003, McCall and Irwin 2006, Thompson and Fernandez 2006). Therefore, understanding the joint action of mutualists and antagonists at the plant individual level is crucial to clarify the costs and benefits of interactions in terms of female reproductive success (FRS hereafter).

Addressing the complexity in plant-animal interactions, and their consequences for individual plant fitness, 
can be achieved by the identification of interaction typologies describing different modes of interaction (Milo et al. 2002, Jordano 2010, Bascompte and Jordano 2014) (Fig. 1a). The study of recurring interaction typologies, called motifs, has been used to analyze a variety of complex webs of interaction (Milo et al. 2002). A gradient of interaction typologies can thus be defined, ranging from an extreme represented by individual plants with fully mutualistic interactions, to the other extreme with fully antagonistic interactions (Fig. 1b).

Our work represents a first attempt to empirically quantify the diversity of plant-animal interactions across individual plants, and their functional effects measured as reproductive outcomes. We hypothesize that such a quantitative typification of distinct interaction typologies based on the sign (mutualistic vs. antagonistic) and strength (weak vs. strong) of animal-plant relationships will have a functional correlate in terms of female reproductive success in plants. Besides, we expected than the increase of mutualistic interaction strength would enhance the contribution to overall pollen transfer within the mating network. To test these hypotheses, we use applications of complex network theory to the pollination of Isoplexis canariensis (L.) J. W. Loudon (Plantaginaceae) (1) to characterize individual-based interaction typologies emerging from the interactions of individual plants with mutualistic and antagonist agents, (2) to estimate the relationship between interaction typology and FRS, (3) to document pollinator movement patterns to infer the relative importance of each plant in the mating network, and (4) to describe the relationship between the relative position in the mating network with the interaction typology and FRS.

\section{Methods \\ Biological system}

We focus on mutualistic and antagonistic agents interacting with the perennial shrub Isoplexis canariensis, a hermaphrodite and self-compatible ornithophilous species endemic to the Canary Islands (Valido et al. 2004). Opportunistic nectar-feeding passerine birds and lacertid lizards act as effective pollinators that increase FRS despite the potential for spontaneous autogamy (Rodríguez-Rodríguez and Valido 2008, RodríguezRodríguez et al. 2013). However, the benefits provided by these vertebrate mutualists are counteracted by invertebrate antagonists (florivores, nectar larcenists and predispersal seed predators), which consume and damage reproductive structures (see Appendix S1: Table S1 for a species list).

\section{Study site}

The study area is located in Teno Alto (Teno Rural Park, NW Tenerife), at an elevation of $870 \mathrm{~m}$ a.s.l. The site is covered by "fayal-brezal" forest with Erica arborea
L. (Ericaceae), Morella faya (Aiton) Wilbur (Myricaceae) and Ilex canariensis Poiret (Aquifoliaceae) as dominant tree species. Populations of I. canariensis are generally found in highly aggregated patches on sun-exposed canopy gaps caused by falling trees, rock outcrops or abandoned agricultural lands. Within this forest we selected two representative, close patches as replicate plots with $67(8.9 \times 13.7 \mathrm{~m})$ and $52(17.5 \times 19.70 \mathrm{~m})$ adult plants each. We monitored tagged individuals during the flowering season (May-September 2008) until just prior to the dispersal of seeds (see Rodríguez-Rodríguez et al. 2015 for details).

\section{Plant-animal interaction typologies}

We grouped animal assemblages into two functional categories: mutualists ( $M$; bird and lizard pollinators), and antagonists ( $A$; floral herbivores, nectar larcenists, and predispersal seed predators; Appendix S1: Table S1). For each individual plant, we defined the interaction strength as the frequency of interactions of specific animal groups with the plant, multiplied by the intensity of interaction. For mutualists, we estimated the interaction frequency as the proportion of censuses in which the plant-pollinator interaction was observed, and the intensity of interaction as the average proportion of flowers probed per plant visit. For antagonists, we estimated the frequency of interaction as the proportion of plant surveys in which we observed signs of damage, and the intensity of interaction as the average proportion of damaged reproductive units per plant survey. We defined the mutualistic interaction strength for the individual plant as the sum of the interactions with bird and lizard, and the antagonistic interaction strength as the sum of interactions with floral herbivores, nectar larcenists and predispersal seed predators. Both mutualistic and antagonistic interaction strengths were standardized (see Rodríguez-Rodríguez et al. 2015 and Appendix S2).

We used the interaction strengths to obtain a quantitative representation of interaction typologies based on an adjacency matrix among 119 plants $(P)$ as rows, and two animal groups, mutualists $(M)$ and antagonists $(A)$ as columns. The matrix elements $a_{P, M}$ (individual plant - mutualists) or $a_{P, A}$ (individual plant—antagonists) were $>0$ if we detected any plant-animal interaction, and 0 otherwise. If the interaction occurred, we used the median values of the respective interaction strength as a cut-off to categorize into "weak" ( $a_{P, M}$ or $a_{P, A}<$ median) or "strong" ( $a_{P, M}$ or $a_{P, A} \geq$ median). We deconstructed the bipartite network into its constituent subgraphs to sort out the individual-based interaction patterns. These patterns were illustrated as undirected three-node subgraphs (triads), where each node represents an interacting partner (i.e., individual plant, mutualists, antagonists), and the links connecting nodes illustrate the interaction strengths between the plant and the respective animal group. We obtained $3 \times 3$ possible combinations of interaction with mutualists and/or antagonists resulting in 


\section{Mutualistic interaction}

(a)

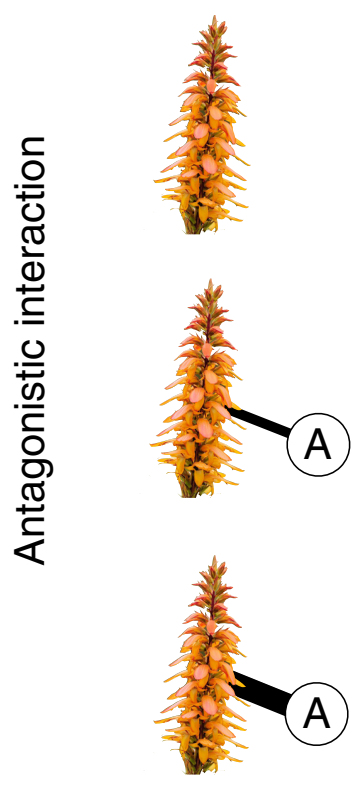

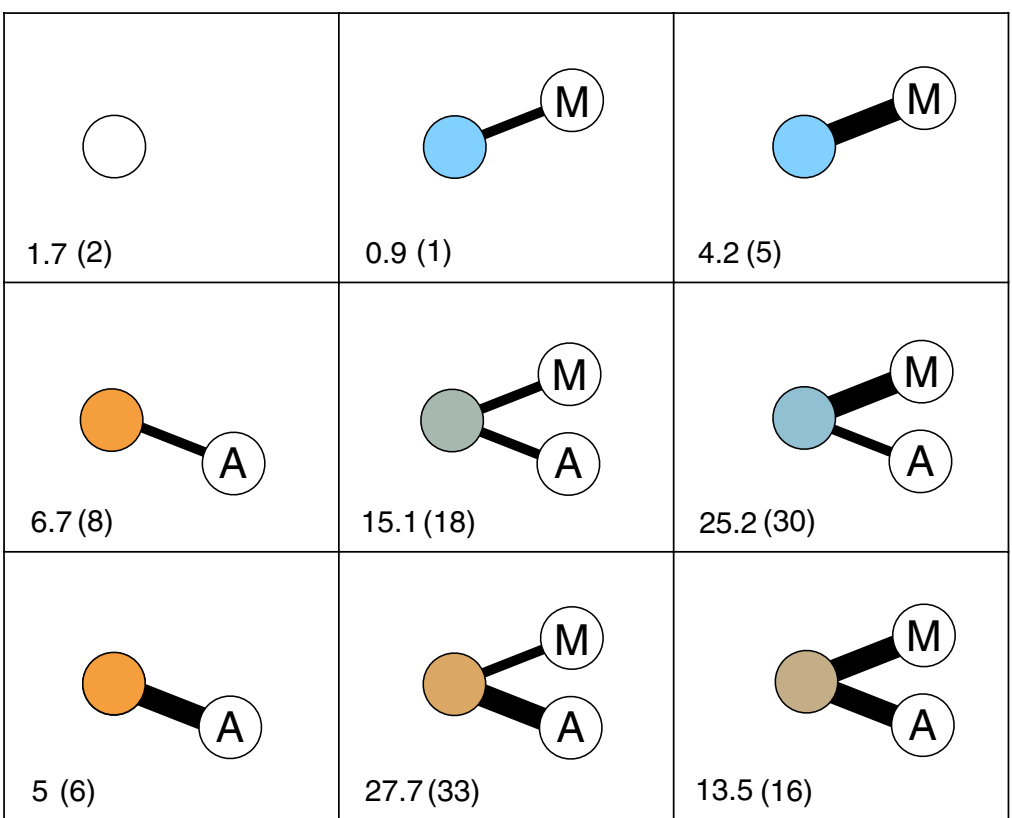
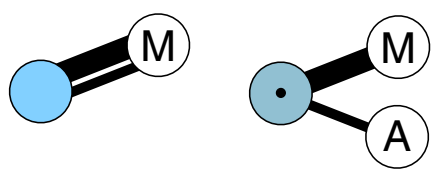

\section{Strong $M$ Weak $A$}

(6)
(30)

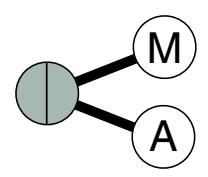

Weak M
Weak A

(18)

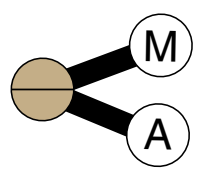

\section{Strong M Strong A}

(16)
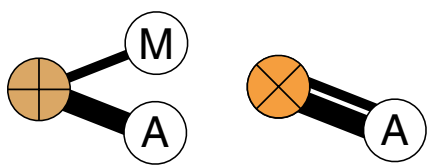

Only A

FIG. 1. Interaction modes between Isoplexis canariensis and mutualistic $M$ and antagonistic $A$ partners. Colors code groups of plants belonging to the same interaction typology (applicable to all figures). (a) The nine interaction triads based on the $3 \times 3$ possible combinations of mutualistic-antagonistic alternatives of interaction modes. Thick lines indicate strong interactions, while narrow lines depict weak interactions. Numbers in the lower-left corner of cells indicate the percentage (sample size in parentheses) of individual plants belonging to each triad (pooling all plants from the two studied patches). The two plants for which we did not record any interaction were later excluded (in white). (b) The six types of interaction typologies organized from the mutualistic to the antagonistic extremes. In the middle, those plants interacting with both animal groups ("diversified" interaction typologies) with all possible combinations of interaction strengths. Each interaction typology is accompanied by its respective label and observed frequency in parentheses (see Appendix S1: Table S1 for species assemblage including in the mutualist and antagonist groups). [Colour figure can be viewed at wileyonlinelibrary.com] 
nine types of triads (Fig. 1a). We recorded the number of plant occurrences in each typology to obtain the observed frequencies. Two plants with no interaction records were excluded from analyses (final $n=117$ ).

\section{Inferred plant contribution to the mating network}

The relative contribution of individual plants to local pollen transfer was calculated in terms of their centrality within the mating network. The centrality of a node (plant) in the complex network of mating events is a measure of its relative importance within the graph (Freeman 1979), i.e., its potential role as a pollen source and/or pollen receiver. Plant centrality was estimated from unipartite weighted networks (one per patch) of individual plants connected by mating events. These mating events were inferred from the sequences of interplant movement by individual bird pollinators within the patch, recorded from direct field observations (Appendix $\mathrm{S} 2$ ). We assume these movements are surrogate indicators of actual events of pollen transfer, a safe assumption given the pollination effectiveness of flower visitation by the birds obtained from previous field experiments (Rodríguez-Rodríguez and Valido 2008, RodríguezRodríguez et al. 2013). We focused on birds, instead of lizards, because they do the majority $(>90 \%)$ of pollinator visits. Previous field experiments have also shown that visits by birds result in most cases in successful pollen deposition and removal; thus, visit and movement records of birds are a proxy to assess actual mating events.

The compiled information from all individual bird movements ( $n=172$ pooling from the two studied patches) was used to estimate link between nodes in the interaction network. Link width was proportional to the frequency of bird movements between each pair of plants, whether the plants acted as pollen donors or sinks (i.e., the bird moved from plant $A-B$ or vice versa). Although selfing events have relevant consequences for FRS (Rodríguez-Rodríguez and Valido 2008), here we focused only on among-plant interactions and, therefore, our networks showed no link connecting a plant to itself. Mating interactions were later represented as adjacency matrices with size $P \times P$, with the individual plants $(P)$ from the same patch as rows $(i)$ and columns $(j)$. If the mating event occurred, $a_{i j}$ values represented the number of events recorded between the specific pair of plants.

We characterized the role of each plant in the mating network by means of its centrality. Centrality indicates how well connected a given individual plant is (in terms of pollen transfer/receipt) to others into the patch as a function of the number of mating events they have shared. Specifically, we used the closeness metric $C$ to estimate plant centrality by using tnet package in $\mathrm{R}$ (Opsahl 2015). This metric is positively related to the shortest number of direct and indirect interactions between one node and all other nodes in a network (shortest paths). $C$ values specify which nodes can be reached fastest from any other node within the network because it interacts with any node using no or few intermediaries (Freeman 1979). In our system where bird movements between plants determine path distance, the metric $C$ would measure how well pollen originating from a plant reaches other plants via the shortest bird flights, or vice versa (i.e., how well pollen from conspecifics reaches the specific plant). We estimated weighted $C$ measures to take into account both the number of intermediary nodes and the link weights, i.e., the number of mating events between any pair of plants (tuning parameter $\alpha=0.5)$ (Opsahl et al. 2010). Thus, links with larger weights were considered to have a much greater impact than links with smaller weights in the mating network. Finally, we normalized closeness measures dividing $C$ by $N-1$, where $N$ is the number of plants in the corresponding patch, to have comparable values across patches.

\section{Female reproductive success}

We estimated FRS of each individual plant as the product of two components: fruit set and viable seed set per fruit. Fruit set was estimated as the proportion of monitored floral pedicels that set fully developed fruits ( $n=8,452$ floral pedicels, range: $8-606$ pedicels/plant). The viable seed set was estimated as the average proportion of viable seeds produced per fruit. We chose a random subset of fruits for each individual plant from similar basal positions in inflorescences to avoid bias caused by maternal effects ( $n=2,026$, range: $3-23$ fruits/plant), and counted the number of viable and aborted seeds inside. Then, we calculated the proportion of seeds within the fruit $\left(P_{\mathrm{vs}}\right)$. Finally, we obtained viable seed set per fruit as the averaged $P_{\text {vs }}$ calculated across all fruits analyzed for each plant (see Rodríguez-Rodríguez and Valido 2008, Rodríguez-Rodríguez et al. 2013, 2015 for details).

\section{Data analysis}

Identification of interaction typologies.-Based on the sign (mutualistic vs. antagonistic) and strength (weak vs. strong) of interactions (Fig. 1a) we tested whether typologies appeared more frequently than expected in a randomized network of the same size. Interaction typologies with observed frequencies above random expectation are known as motifs (Milo et al. 2002). To generate a random network, we resampled $(9,999$ times) the three alternatives of plant-animal interaction strengths (absent, weak, strong), keeping the probabilities of each of these alternatives found in the study population $(0.10,0.45,0.45$, respectively). Once the random network was built, we counted the number of each of the six predefined plantanimal interaction typologies (Fig. 1b) rendered by the iterated matrix following the procedure described in the Plant-animal interaction typologies section. We created six distributions of expected frequencies, which were later used to detect over-represented typologies (i.e., motifs) by one-tailed $Z$ tests. 
Reproductive consequences of interaction typologies. The effect of the interaction typology on FRS was tested using generalized linear regression models (GLM; quasibinomial errors), with FRS as the response variable and plant-animal interaction typology as the explanatory variable. To control for potential external confounding factors, we also included a set of plant traits (Appendix S3), and patch identity as fixed effects. Prior to model fitting, plant traits were standardized and tested for multi-collinearity via Variance Inflation Factors (VIF $<2$ for all predictor variables; $\mathrm{HH}$ package in R) (Heiberger 2013). The significance of regressors was tested by comparing the full model with models that excluded only the specific factor by means of likelihood ratio tests (Zuur et al. 2009). The relative weight of each regressor was assessed with the relaimpo package in $\mathrm{R}$ (Groemping 2006), which estimates relative importance of regressors by the $R^{2}$ contribution averaged over resampled orderings among regressors. Given that relaimpo only implements models with Gaussian errors, we also compared the standardized coefficients by expressing each as a percentage of the total sum of the absolute values of the estimates. This is an approximation to assess the relative importance of effects in GLM models with non-Gaussian errors. Finally, we tested for differences in FRS among interaction typologies by post-hoc pairwise comparisons. The comparisons were done with adjusted probability values by the single step method by using the $\mathrm{R}$ package multcomp (Hothorn et al. 2008).

Mating consequences of interaction typologies.-To establish the functional consequences of pollen transfer by birds' movements, we first explored the relationship between the closeness of each individual plant with their FRS. For this, we tested their relationships by GLM (quasibinomial errors). Again, we standardized plant traits and patch identity as fixed effects to control for potential confounding factors, and tested the significance of regressors by means of likelihood ratio tests. Secondly, we obtained the grand means of $C$ and FRS per plant-animal interaction typology to explore graphically if interaction typologies with higher levels of mutualism were consistently associated with higher values of closeness and female reproductive outcome.

All analyses and graphical representations were generated with $\mathrm{R}$ software version 3.1.1 ( $\mathrm{R}$ Development Core Team 2014).

\section{RESULTS \\ Interaction typologies}

We identified six interaction typologies of plant-animal interactions based on the interaction sign and strengths for mutualists $(M)$ and antagonists $(A)$ (Fig. 1b). The resulting triads simplified the complexity of interaction patterns found along the mutualism-antagonism gradient for the large number of individual plants studied.
One interaction typology, i.e., motif-plants that interacted weakly with mutualists but strongly with antagonists ("Weak M-Strong A") — was recorded significantly more frequently than expected by chance $(Z=2.16$, $P=0.02$, Fig. 2a). In addition, plants that interacted strongly with mutualists but weakly with antagonists ("Strong M-Weak A") were also marginally overrepresented $(Z=1.46, P=0.07)$.

\section{Reproductive consequences of interaction typologies}

Female plant reproductive success was significantly associated with the interaction typology the plant belonged to (Table 1, Fig. 2b; see Appendix S4: Table S1A). This effect was significant even when statistically controlling for the rest of covariates, which did not have a significant effect on FRS $(P>0.05$ in all cases; see Appendix S4: Table S1B).

We found a decreasing trend in female fitness from fully mutualistic to fully antagonistic interaction typologies (from left to right in Fig. 2b; see Appendix S4: Table S1A). Plants that interacted exclusively with mutualists ("Only M"), or interacted with both animal groups but weakly with antagonists ("Strong M-Weak A", "Weak M-Weak A") had similarly high reproductive outcomes $(P>0.05$ for all pairwise contrasts; grouped by letter $a$ in Fig. 2 b; see also Appendix S4: Table S1A). Conversely, those plants that interacted only with antagonists ("Only A"), or interacted strongly with antagonists independent of their interaction strength with mutualists ("Strong M-Strong A", "Weak M-Strong A") showed similarly low reproductive success $(P>0.05$ for all pairwise contrasts; grouped by letter $c$ in Fig. 2b). The continuous variation in FRS shows that the combination of mutualistic and antagonistic interaction strengths is more accurate at predicting reproductive consequences when compared to simpler typologies. For example, mutualistic interactions explain just $23.14 \%$ of variation in FRS. By far, the largest relative effect on FRS is due to antagonist agents $(68.85 \%)$ with floral herbivores contributing a $39.83 \%$ of the total (Appendix S5).

Thus, the quantitative typification of distinct interaction typologies based on the sign (mutualistic vs. antagonistic) and strength (weak vs. strong) of animal-plant relationships helps summarize the broad diversity of interaction "modes" occurring in nature, very often showing extreme complexity. In this way, the variation across interaction typologies (Fig. 2) adequately captured the continuous, quantitative effects of animal partners (Appendix S5). Overall, the analysis of FRS variation in relation to continuous values of interaction strengths with different groups of organisms supports the conclusions based on interaction typologies (see Results in Appendix S5).

\section{Mating consequences of interaction typologies}

The heterogeneous distribution of bird movements among plants within each patch resulted in contrasting 

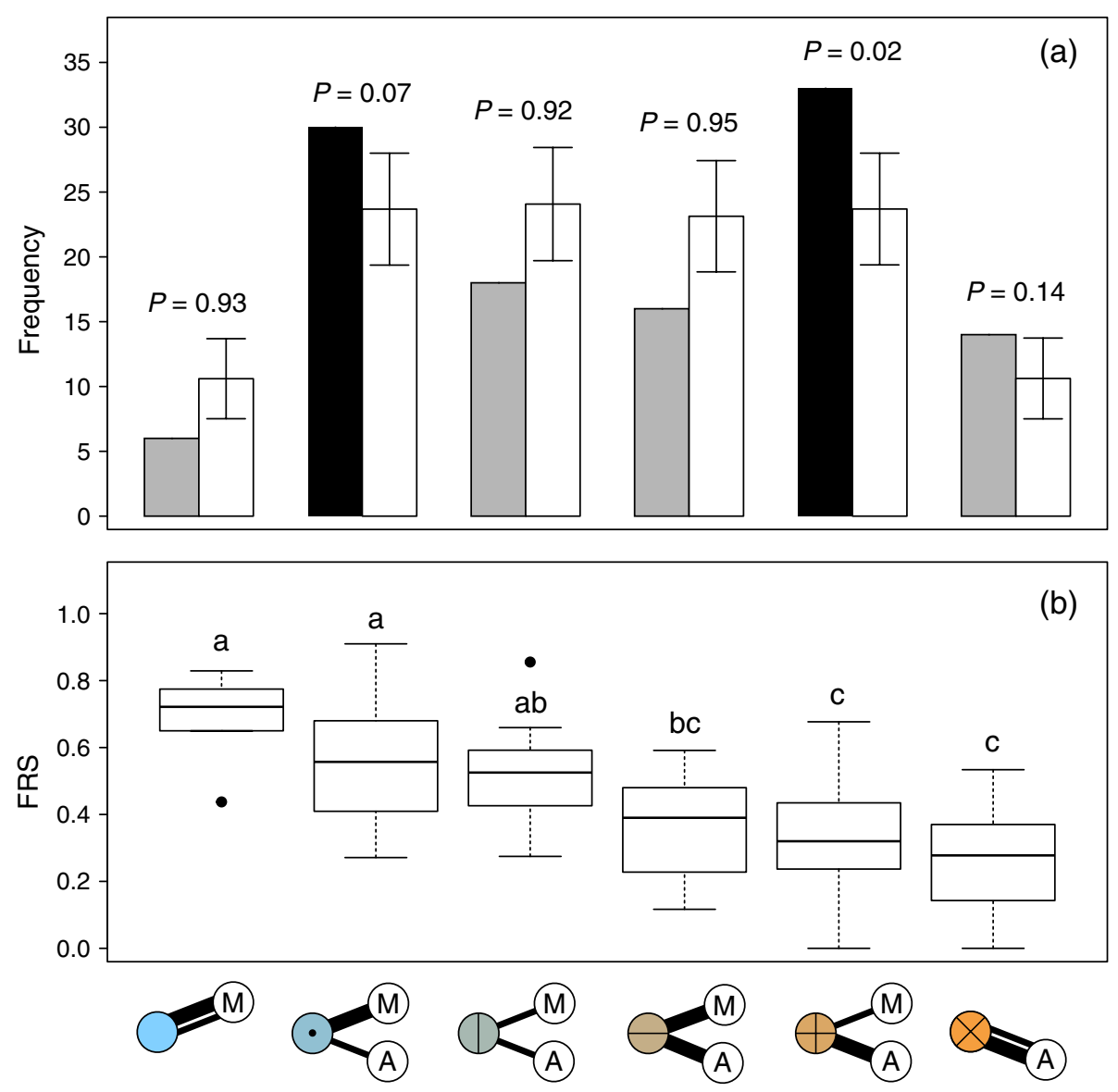

FIG. 2. Identification of plant-animal interaction typologies in the study system and their reproductive outcomes. (a) Observed (filled) and expected (unfilled) frequencies of the six interaction typologies. Mean $\pm \mathrm{SD}$ are represented for expected values. $P$ values were obtained from upper-tailed $Z$ tests $(\alpha=0.05$ ). Black bars indicate identified interaction motifs (i.e., overrepresented). (b) Effect of the interaction typology on female reproductive success (FRS) analyzed by linear regression. FRS was defined as fruit set multiplied by viable seed set per fruit. Different letters indicate statistical differences in reproductive outcomes among interaction typologies $(P<0.05$; see post-hoc multiple comparisons in Appendix S4). [Colour figure can be viewed at wileyonlinelibrary.com]

topological positions of individual plants in the inferred mating network (Fig. 3). On average, plants occupied positions of moderate closeness $(C=0.29 \pm 0.21$, range $=0-0.72, n=117$ plants). However, we found ample variation ranging from plants completely disconnected from the mating dynamics $(C=0, n=34)$ to others with high integration $(C>0.60$, range $=0.64-0.72, n=5$; Fig. 3).

Plant closeness had a significant effect on FRS: plants with higher $C$ values had higher female fitness (Fig. 4; Appendix S6). The increase in plant closeness and associated reproductive benefits partially correlated with a transition from fully mutualistic to fully antagonistic interactions (Fig. 4). Hence, individual plants interacting exclusively with antagonists were totally decoupled from the mating network and showed the lowest reproductive outcomes ("Only A", $C=0$ for all plants, $n=14$ ). However, increasing plant closeness and reproductive success were not associated with a decrease in antagonistic effects and a related increase in mutualistic interactions. This was contrary to our expectation of highest $C$ and FRS values in plants visited exclusively by mutualists. In contrast, the highest values of closeness were found in those plants that interacted with both functional groups: strongly with mutualists and weakly/strongly with antagonists (average $C=0.43 \pm 0.15$, range $=0-0.72$, $n=46$ ). With intermediate values, we found plants that interacted exclusively with mutualists, or weakly with mutualists and weakly/strongly with antagonists (average $C=0.24 \pm 0.19$, range $=0-0.59, n=57$; Fig. 4)

\section{Discussion}

Studies addressing plant-animal mutualistic networks have provided novel insights on their functional consequences for natural communities (Bascompte and Jordano 2014). It has, however, been challenging to understand (1) how merging mutualistic and antagonistic interactions into a single network alters these consequences in terms of plant reproductive success (Fontaine et al. 2011), and (2) how these consequences are driven by the heterogeneous distribution of interactions at lower 
TABLE 1. Summary of the generalized linear regression model showing the effects of plant-animal interaction typology, patch identity and standardized plant traits on female reproductive success (FRS)

\begin{tabular}{lccc}
\hline \hline Regression coefficients & Estimate \pm SE & $t$ Value & $P$ value \\
\hline Intercept & $0.282 \pm 0.404$ & 0.70 & 0.48 \\
Interaction typology "Only antagonists" & $-1.749 \pm 0.374$ & -4.68 & $<0.001$ \\
Interaction typology "Weak M-Weak A" & $-0.664 \pm 0.346$ & -1.92 & 0.05 \\
Interaction typology "Strong M-Weak A" & $-0.564 \pm 0.342$ & -1.65 & 0.10 \\
Interaction typology "Weak M-Strong A" & $-1.415 \pm 0.341$ & -4.15 & $<0.001$ \\
Interaction typology "Strong M-Strong A" & $-1.202 \pm 0.363$ & -3.31 & $<0.001$ \\
Plant height & $-0.074 \pm 0.069$ & -1.07 & 0.28 \\
Nectar sugar reward & $-0.022 \pm 0.068$ & -0.32 & 0.75 \\
Flowering synchrony & $-0.032 \pm 0.086$ & -0.37 & 0.71 \\
Number of neighbours in $r=2$ m & $0.051 \pm 0.092$ & 0.56 & 0.58 \\
Distance to the nearest tree & $-0.063 \pm 0.084$ & -0.75 & 0.45 \\
Patch & $0.307 \pm 0.186$ & 1.65 & 0.10 \\
\hline
\end{tabular}

Notes: FRS was estimated as the product of the proportion of fruits produced per plant and the averaged proportion of viable seeds per fruit (see Methods: Female reproductive success). Significance of regressors was tested by likelihood ratio tests (see also Appendix S4). Only interaction topology resulted in a significant effect (Deviance $=7.44$, $\mathrm{df}=5, P<0.001$ ).

organizational levels, i.e., among individuals within populations (Poisot et al. 2015). We address this challenge with new methods to characterize the intraspecific distribution of plant-animal interactions along the mutualisticantagonistic gradient, and its functional correlates with plant reproductive success. Our study shows that even at small population scales, interacting animal assemblages vary considerably across individual plants, independently of spatial autocorrelation effects (Rodríguez-Rodríguez et al. 2015). However, this variation is not randomly distributed within the population, as illustrated by the presence of interaction motifs. Here, we find that the joint effects of mutualists and antagonists, depending on the relative interaction strengths, leave a signal in the relative contribution of plants as sources of viable propagules for the next generation. As expected, female reproductive success decreases from the fully mutualistic extreme to the fully antagonistic extreme of the interaction gradient. However, the existence of antagonistic damage, combined with strong mutualistic interactions, increases the participation of plants in the local dynamics of pollen transfer. Therefore, quantifying the strength of plantanimal interactions, rather than solely recording their presence or absence, improves our predictions of plant fitness and understanding of population dynamics.

\section{Interaction typologies}

Most plants involved in this study have in common that their animal assemblages include both mutualists and antagonists, but with variable interaction strengths. That is, if the strength of either type of interaction is strong, then the other type is weak. The two detected interaction motifs were "Weak M-Strong A" and "Strong M-Weak A", significantly or marginally overrepresented, respectively (Fig. 2a). At this point, one might ask what drivers restrict individual plants from interacting with similar strengths with both functional groups. In our system, we can reasonably explain this by considering the indirect effects of the interacting animal groups on each other. For example, it is known that pollinators are able to discriminate between damaged plants by antagonists (Irwin and Brody 1998, Krupnick et al. 1999, Carper et al. 2016). Alternatively, antagonists can also detect and reject plants depleted of floral resources by mutualists (Stout et al. 1998). The recognition of each other's action results in a high frequency of plants that are greatly benefited from mutualists and poorly damaged by antagonists, and vice versa. In the case of Isoplexis canariensis, florivores seem to be the main drivers of the observed asymmetrical typologies because they interact with most plants in the population, and their action occurs even before the activity of pollinators. The early effect of florivores has great potential to influence the later movements of pollinators (i.e., the mating network), an explanation also supported by the negative spatial correlation between mutualistic and antagonistic interactions previously found in the two studied patches (Rodríguez-Rodríguez et al. 2015, see also Appendix S5).

Theoretical studies that have analyzed ecological networks with diversified interactions suggest that asymmetrical interactions may be prevalent in nature because of their associated benefits (Melián et al. 2009, Mougi and Kondoh 2012, 2014). First, the moderate mixture of mutualistic and antagonistic interactions gives greater stability and persistence to plant populations that scales up to maintain communities (Mougi and Kondoh 2012, 2014). Our divergent typologies assure the coexistence of mutualists and antagonists given that they appear in similar proportions (Fig. 2a). According to these theoretical approaches, stability drastically decreases when there is a skew towards either interaction type (Mougi and Kondoh 2012, 2014), a fact that may explain the relatively lower occurrence of plants that interact with a 


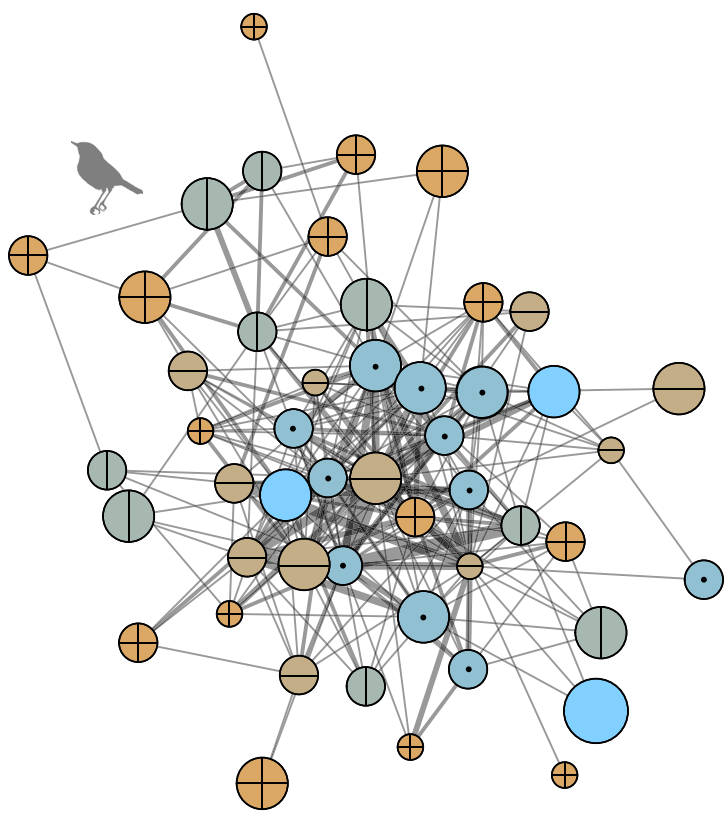

Patch 1

$$
\begin{gathered}
C=0.33 \pm 0.22 \\
(0-0.72) \\
49(17)
\end{gathered}
$$

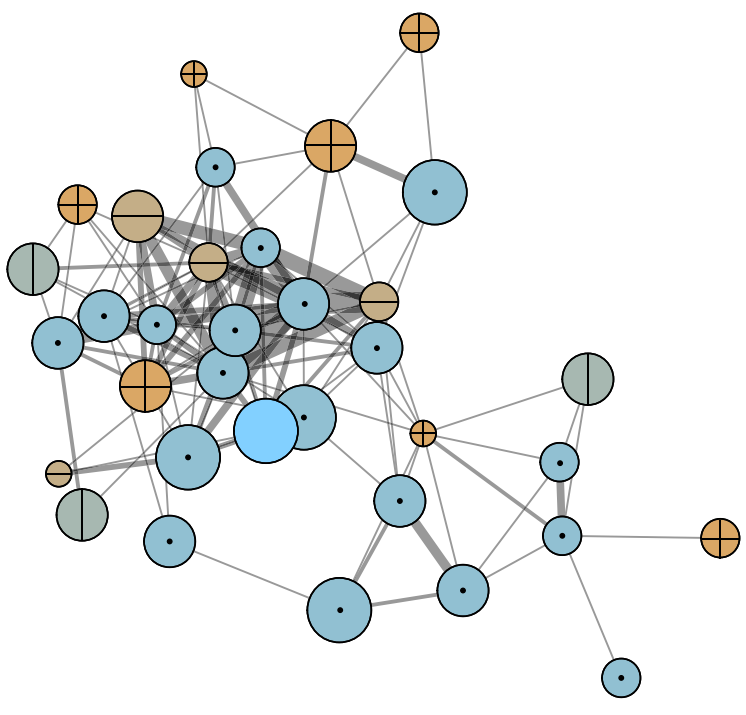

Patch 2

$$
\begin{gathered}
C=0.23 \pm 0.19 \\
(0-0.64) \\
34(17)
\end{gathered}
$$

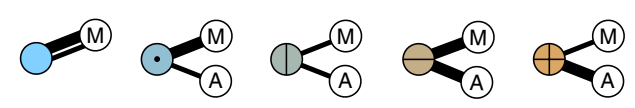

FIG. 3. Unipartite networks illustrating the inferred mating patterns (i.e., potential pollen transfer events) among individual plants originating from the $n=172$ bird pollinator foraging movements. The links among nodes (plants) indicate the existence of at least one observed mating event between any pair of plants. Link width is proportional to the observed number of mating events at the patch scale. Node size refers to the female reproductive success (FRS) achieved by the plant, which was categorized into five levels for graphical representation: (1) FRS $=0$, (2) $0<\mathrm{FRS} \leq 0.25$, (3) $0.25<\mathrm{FRS} \leq 0.50$, (4) $0.50<\mathrm{FRS} \leq 0.75$, (5) $0.75<\mathrm{FRS} \leq 1$. Network representation was generated with the Kamada-Kawai energy-minimization algorithm (Kamada and Kawai 1989). For each patch, we indicate mean \pm SD of closeness (range in parentheses), and below, the number of connected and disconnected plants (in parentheses). [Colour figure can be viewed at wileyonlinelibrary.com]

single functional group (Fig. 2a). Second, the prevalence of asymmetrical interactions favors the maintenance of more complex biodiversity compared to populations with simpler typologies (Mougi and Kondoh 2012). Higher complexity increases the diversity of plant reproductive outcomes, which ultimately boosts variation fueling coevolutionary processes (Thompson 2005).

\section{Reproductive consequences of interaction typologies}

As expected, the predefined categories of distinct plantanimal interaction typologies along the mutualismantagonism gradient correlated with individual plant female reproductive success. The fact that we consistently found a significant effect of interaction typology on FRS suggests that the combination of mutualistic and antagonistic interaction strengths within individual plants drives variation in FRS beyond the effects of plant traits and patch identity.
The continuous variation in FRS shows that the combination of mutualistic and antagonistic interaction strengths more accurately predicts reproductive consequences when compared to simpler typologies. Although the interannual consistency of this pattern needs to be evaluated, our results reveal that, even at the scale of small populations, the reproductive outcomes of plantpollinator interactions are highly dynamic due to variation in antagonistic interactions (Irwin and Brody 1998, Krupnick et al. 1999, Herrera et al. 2002, Bronstein et al. 2003, Strauss and Irwin 2004, Thompson and Fernandez 2006).

\section{Mating consequences of interaction typologies}

Contrary to our expectations, plants with fully legitimate mutualistic interactions did not have the highest closeness values (did not contribute the most pollen transfer) despite having the highest female reproductive 


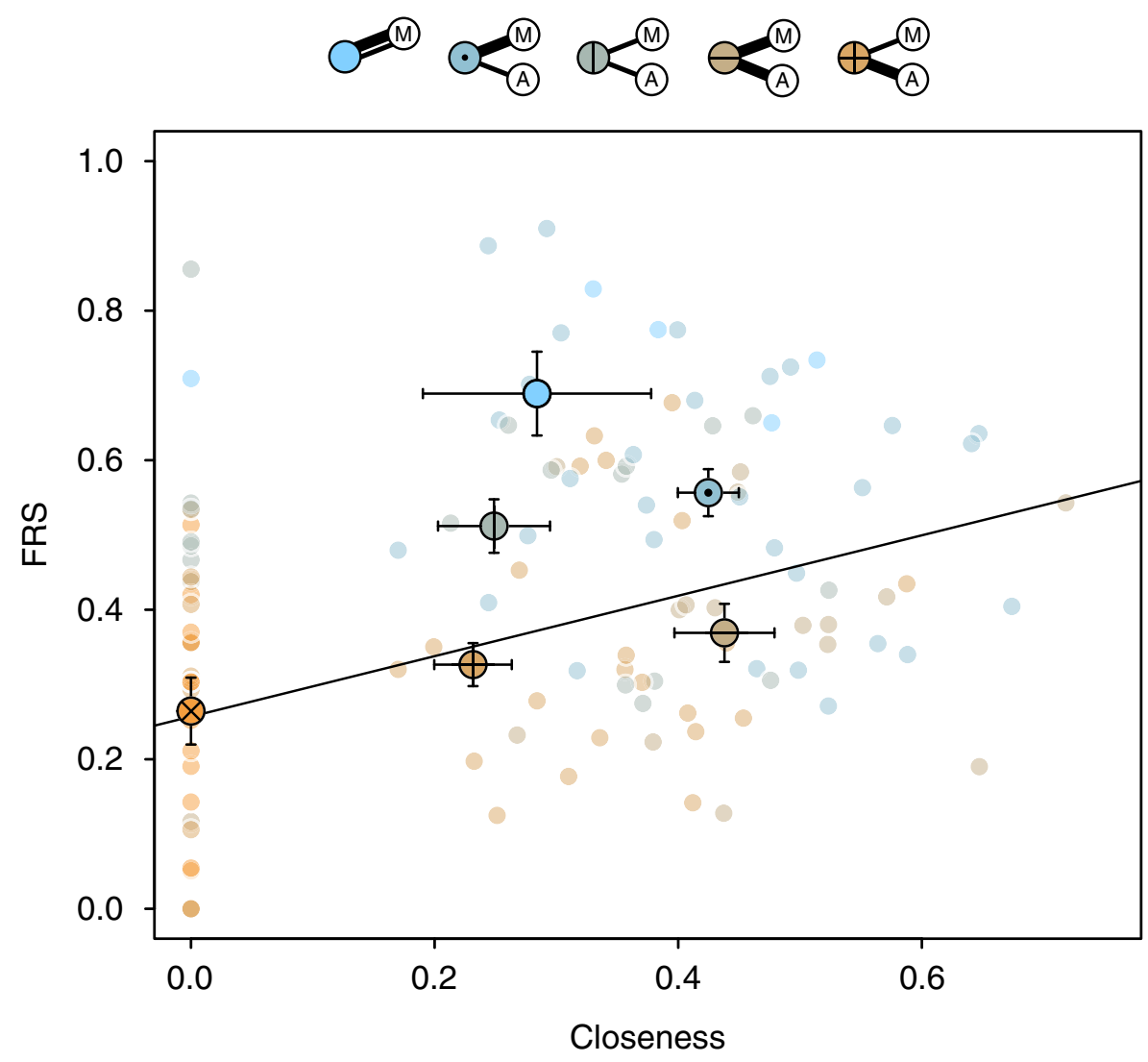

FIG. 4. Relationship between the centrality (closeness) of individual plants in the mating network and associated female reproductive success (FRS). Larger, plotted dots are group means \pm SE per plant-animal interaction typology. Smaller dots at the background represent values of individual plants (total $n=117$ ). Model intercept and slope ( $\beta$ coefficient of closeness $C$ ) are given in Appendix S6. Averaged means \pm SD of closeness and fitness ( $C$, FRS) per interaction typology: "Only M" $(C=0.28 \pm 0.23$, FRS $=0.69 \pm 0.14)$; "Strong M-Weak A": $(C=0.42 \pm 0.14$, FRS $=0.56 \pm 0.17)$; "Weak M-Weak A": $(C=0.25 \pm 0.19$, FRS $=0.51 \pm 0.15)$; "Strong M-Strong A": $(C=0.44 \pm 0.16$, FRS $=0.37 \pm 0.16)$; "Weak M-Strong A": $(C=0.23 \pm 0.18$, FRS $=0.33 \pm 0.17)$; and "Only A": $(C=0, \mathrm{FRS}=0.26 \pm 0.17)$. [Colour figure can be viewed at wileyonlinelibrary.com]

success. In contrast, plants showing strong mutualistic interactions, but also (weak or strong) interactions with antagonists, occupied the most central positions in the mating network (i.e., higher closeness; Figs. 3 and 4). Thus, the presence of antagonistic interactions does not imply reproductive isolation within the mating network, but gives plants the potential to participate in the pollen flow and increase both female and male fitness. Our findings indicate that antagonistic interactions may indirectly promote outcrossing and increased pollen export/ reception for individual plants.

The higher closeness values of plants with mixed mutualistic-antagonistic interactions may be due to individual plant characteristics, such as larger floral displays compared to other conspecifics $(P<0.05$, Appendix S7; see also Gómez and Perfectti 2012, Dupont et al. 2014). These plant traits are especially attractive to both pollinators and antagonists causing conflicting reproductive pressures (Ågren et al. 2013). Here we propose an alternative, non-exclusive, hypothesis: the presence of antagonistic damage enhances plant centrality in the mating network (Fig. 4). For example, the most central plants with diversified interactions incorporating antagonists ("Strong M-Weak A", "Strong M-Strong A") have higher levels of florivory and nectar larceny than less central plants with fully mutualistic interactions ("Only M"), which have no damage at all. Probably attracted by the large floral displays of these central plants, pollinators may detect partial floral damage during the visit, and/or reduced nectar rewards, and probe fewer flowers in less time compared to undamaged plants (Zimmerman and Cook 1985, Irwin and Brody 1998, Irwin 2003). These aspects may force pollinators to move more often among plants (Maloof and Inouye 2000).

Despite the higher closeness values of individual plants with diversified animal assemblages, their reproductive outcomes are lower compared to fully mutualistic typologies (Fig. 4). Female reproductive success is markedly reduced under strong antagonistic interactions, an obvious result if we consider that floral herbivores and seed predators consume pollen grains, ovules and seeds. The effect of antagonists can also reduce female fitness via 
pollinators' behavioural changes since they are less frequently visited by pollinators, which probe a lower proportion of flowers and deposit less pollen (Thomson and Plowright 1980, Zimmerman and Cook 1985, Irwin and Brody 1998, Krupnick et al. 1999, Irwin 2003, McCall and Irwin 2006, Carper et al. 2016). However, centrality may enhance plant reproductive success in other aspects not evaluated in the present study. For example, the higher integration of plants in the mating network may lead to increased outcrossing. However, the relationship between floral damage and pollinator visitation is complex and unfortunately, we know relatively little about how antagonists may promote pollen and gene flow (Krupnick et al. 1999, McCall and Irwin 2006, Carper et al. 2016). Thus, future field experiments including paternal analysis of offspring to infer pollen dispersal patterns will shed light on the role of antagonists in pollination.

\section{Conclusions}

This paper aims to better characterize the complexity of plant-animal interactions occurring in natural populations and their functional consequences for plants (Gómez et al. 2011, Gómez and Perfectti 2012). We found extreme variation in interactions with six typologies identified but just 1-2 motifs, with strong effects on individual FRS. We demonstrate the expected decrease in FRS from mutualistic to antagonistic modes along with the unexpected finding that plants with damage are better connected in the mating network via increased pollen transfer/receipt. However, we realise that our results raise new questions that require further research. The question remains, how interaction typologies (and motifs) vary across space and time. The same species may interact in different ways when their local abundances vary, their trait distribution changes, or when the environment affects either of these factors (Siepielski and Benkman 2010, Ågren et al. 2013, Poisot et al. 2015). Secondly, even in the hypothetical situation that interaction typologies were consistent across plant species, whether they render similar reproductive outcomes for species with different breeding systems is unknown. For example, self-compatible plants can overcome strong antagonistic interactions more easily than self-incompatible plants, which might suffer a greater reduction in pollen transfer and seed production with greater damage. Third, further research in other pollination systems is needed to assess under which circumstances antagonistic damage may promote pollen and gene flow (i.e., outcrossing). Our study opens up a new approach to understanding how the building blocks of plant-animal interactions emerge within populations and, through their functional consequences, drive the dynamics of coevolution.

\section{ACKNOWLEDGMENTS}

We are grateful for comments provided by Francisco Rodríguez-Sánchez and John N. Thompson, technical support by IPNA-CSIC and the city council of Buenavista del Norte (Tenerife), and permits by Cabildo Insular de Tenerife. The manuscript benefited from comments by M. Sinnott-Armstrong, Neal Williams, and two anonymous reviewers. The study was funded by a Predoctoral Fellowship (I3P I3PPRE_06_00019 CSIC) to MCRR, a Postdoctoral Grant (RYC-2007-00620) to AV, and a Junta de Andalucía Excellence Grant (RNM-5731) to PJ, as well as a Severo Ochoa Excellence Award from the Ministerio de Economía y Competitividad (SEV-2012-0262).

\section{Literature Cited}

Ågren, J., F. Hellström, P. Toräng, and J. Ehrlén. 2013. Mutualists and antagonists drive among-population variation in selection and evolution of floral display in a perennial herb. Proceedings of the National Academy of Sciences USA 110:18202-18207.

Bascompte, J., and P. Jordano. 2014. Mutualistic networks. Princeton University Press, Princeton, New Jersey, USA.

Bronstein, J. L., W. G. Wilson, and W. F. Morris. 2003. Ecological dynamics of mutualist/antagonist communities. American Naturalist 162:S24-S39.

Cantor, M., M. M. Pires, G. O. Longo, P. R. Guimarães, and E. Z. F. Setz. 2013. Individual variation in resource use by opossums leading to nested fruit consumption. Oikos 122: 1085-1093.

Carper, A. L., L. S. Adler, and R. E. Irwin. 2016. Effects of florivory on plant-pollinator interactions: implications for male and female components of plant reproduction. American Journal of Botany 103:1061-1070.

Dáttilo, W., R. Fagundes, C. A. Q. Gurka, M. S. A. Silva, M. C. L. Vieira, T. J. Izzo, C. Díaz-Castelazo, K. Del-Claro, and V. Rico-Gray. 2014. Individual-based ant-plant networks: diurnal-nocturnal structure and species-area relationship. PLoS ONE 9:e99838.

Dupont, Y. L., K. Trøjelsgaard, M. Hagen, M. V. Henriksen, J. M. Olesen, N. M. E. Pedersen, and W. D. Kissling. 2014. Spatial structure of an individual-based plant-pollinator network. Oikos 123:1301-1310.

Dupont, Y. L., K. Trøjelsgaard, and J. M. Olesen. 2011. Scaling down from species to individuals: a flower-visitation network between individual honeybees and thistle plants. Oikos 120: 170-177.

Fontaine, C., P. R. Guimarães Jr., S. Kéfi, N. Loeuille, J. Memmott, W. H. van der Putten, F. J. F. van Veen, and E. Thébault. 2011. The ecological and evolutionary implications of merging different types of networks. Ecology Letters 14:1170-1181.

Fortuna, M. A., C. García, P. R. Guimarães Jr., and J. Bascompte. 2008. Spatial mating networks in insectpollinated plants. Ecology Letters 11:490-498.

Freeman, L. C. 1979. Centrality in social networks: conceptual clarification. Social Networks 1:215-239.

Gómez, J. M., and F. Perfectti. 2012. Fitness consequences of centrality in mutualistic individual-based networks. Proceedings of the Royal Society of London B: Biological Sciences 279:1754-1760.

Gómez, J. M., F. Perfectti, and P. Jordano. 2011. The functional consequences of mutualistic network architecture. PLoS ONE 6:e16143.

Groemping, U. 2006. Relative importance for linear regression in R: the package relaimpo. Journal of Statistical Software 17:1-27.

Heiberger, R. M. 2013. HH: statistical analysis and data display: Heiberger and Holland. R package. http://CRAN.R-project. org/package $=\mathrm{HH}$ 
Herrera, C. M., M. Medrano, P. J. Rey, A. M. SánchezLafuente, M. B. García, J. Guitián, and A. J. Manzaneda. 2002. Interaction of pollinators and herbivores on plant fitness suggests a pathway for correlated evolution of mutualism- and antagonism-related traits. Proceedings of the National Academy of Sciences USA 99:16823-16828.

Hothorn, T., F. Bretz, and P. Westfall. 2008. Simultaneous inference in general parametric models. Biometrical Journal 50:346-363.

Irwin, R. E. 2003. Impact of nectar robbing on estimates of pollen flow: conceptual predictions and empirical outcomes. Ecology 84:485-495.

Irwin, R. E., and A. K. Brody. 1998. Nectar robbing in Ipomopsis aggregata: effects on pollinator behavior and plant fitness. Oecologia 116:519-527.

Jordano, P. 2010. Coevolution in multispecific interactions among free-living species. Evolution: Educational and Outreach 3:40-46.

Kamada, T., and S. Kawai. 1989. An algorithm for drawing general undirected graphs. Information Processing Letters 31:7-15.

Kissling, W. D., and M. Schleuning. 2015. Multispecies interactions across trophic levels at macroscales: retrospective and future directions. Ecography 38:346-357.

Krupnick, G. A., A. E. Weis, and D. R. Campbell. 1999. The consequences of floral herbivory for pollinator service to Isomeris arborea. Ecology 80:125-134.

Maloof, J. E., and D. W. Inouye. 2000. Are nectar robbers cheaters or mutualists? Ecology 81:2651-2661.

McCall, A. C., and R. E. Irwin. 2006. Florivory: the intersection of pollination and herbivory. Ecology Letters 9: $1351-1365$.

Melián, C. J., J. Bascompte, P. Jordano, and V. Křivan. 2009. Diversity in a complex ecological network with two interaction types. Oikos 118:122-130.

Milo, R., S. Shen-Orr, S. Itzkovitz, N. Kashtan, D. Chklovskii, and U. Alon. 2002. Network motifs: simple building blocks of complex networks. Science 298:824-827.

Mougi, A., and M. Kondoh. 2012. Diversity of interaction types and ecological community stability. Science 337: 349-351.

Mougi, A., and M. Kondoh. 2014. Instability of a hybrid module of antagonistic and mutualistic interactions. Population Ecology 56:257-263.

Opsahl, T. 2015. Tnet: software for analysis of weighted, twomode, and longitudinal networks. $\mathrm{R}$ package. http:// CRAN.R-project.org/package $=$ Tnet

Opsahl, T., F. Agneessens, and J. Skvoretz. 2010. Node centrality in weighted networks: generalizing degree and shortest paths. Social Networks 32:245-251.
Poisot, T., D. B. Stouffer, and D. Gravel. 2015. Beyond species: Why ecological interaction networks vary through space and time. Oikos 124:243-251.

R Development Core Team. 2014. R: a language and environment for statistical computing, version 3.1.1. R Foundation for Statistical Computing, Vienna, Austria.

Rodríguez-Rodríguez, M. C., P. Jordano, and A. Valido. 2013. Quantity and quality components of effectiveness in insular pollinator assemblages. Oecologia 173:179-190.

Rodríguez-Rodríguez, M. C., P. Jordano, and A. Valido. 2015. Hotspots of damage by antagonists shape the spatial structure of plant-pollinator interactions. Ecology 96:2181-2191.

Rodríguez-Rodríguez, M. C., and A. Valido. 2008. Opportunistic nectar-feeding birds are effective pollinators of birdflowers from Canary Islands: experimental evidence from Isoplexis canariensis (Scrophulariaceae). American Journal of Botany 95:1408-1415.

Siepielski, A. M., and C. W. Benkman. 2010. Conflicting selection from an antagonist and a mutualist enhances phenotypic variation in a plant. Evolution 64:1120-1128.

Stout, J. C., D. Goulson, and J. A. Allen. 1998. Repellent scentmarking of flowers by a guild of foraging bumblebees (Bombus spp.). Behavioral Ecology and Sociobiology 43:317-326.

Strauss, S. Y., and R. E. Irwin. 2004. Ecological and evolutionary consequences of multispecies plant-animal interactions. Annual Review of Ecology, Evolution and Systematics 35: 435-466.

Thompson, J. N. 2005. The geographic mosaic of coevolution. University of Chicago Press, Chicago, Illinois, USA.

Thompson, J. N., and C. C. Fernandez. 2006. Temporal dynamics of antagonism and mutualism in a geographically variable plant-insect interaction. Ecology 87:103-112.

Thomson, J. D., and R. C. Plowright. 1980. Pollen carryover, nectar rewards, and pollinator behavior with special reference to Diervilla lonicera. Oecologia 46:68-74.

Tur, C., J. M. Olesen, and A. Traveset. 2014. Increasing modularity when downscaling networks from species to individuals. Oikos 124:581-592.

Valido, A., Y. L. Dupont, and J. M. Oleen. 2004. Bird-flower interactions in the Macaronesian islands. Journal of Biogeography 31:1945-1953.

Valverde, J., J. M. Gómez, and F. Perfectti. 2016. The temporal dimension in individual-based plant pollination networks. Oikos 125:468-479.

Zimmerman, M., and S. Cook. 1985. Pollinator foraging, experimental nectar-robbing and plant fitness in Impatiens capensis. American Midland Naturalist 113:84-91.

Zuur, A. F., E. N. Ieno, N. J. Walker, A. A. Saveliev, and G. M. Smith. 2009. Mixed effects models and extensions in ecology with R. Springer, New York, New York, USA.

\section{SUPPORTING INFORMATION}

Additional supporting information may be found in the online version of this article at http://onlinelibrary.wiley.com/ doi/10.1002/ecy.1756/suppinfo 